\title{
Clindamycin concentrations in umbilical cord blood after maternal exposure: a pooled analysis.
}

\section{Karel Allegaert}

Department of Pediatrics, Division of Neonatology, Erasmus MC-Sophia Children's Hospital, the Netherlands, k.allegaert@erasmusmc.nl, and Department of Development and Regeneration, KU Leuven, Belgium, karel.allegaert@uzleuven.be

\section{Background and introduction}

In the presence of maternal allergy to cephalosporins or because of center-specific guidelines, clindamycin is used in women at delivery. We aimed to summarize the reported observations on clindamycin concentrations in umbilical cord blood (UCB) after maternal exposure.

\section{Methods}

Using a systematic PubMed search, we retrieved 4 publications that reported on maternal-UCB concentrations during either single or repeated clindamycin (450 to $900 \mathrm{mg}$ ) iv administration [Muller et al, Antimicrob Agents Chemother 2010; Wear et al, J Perinatol 2016; Philipson et al, NEJM 1973; Weinstein et al, Am J Obstet Gynecol 1976].
Results

Based on 33 observations, the mean concentration observed in UCB was $2.78 \mathrm{mg} / \mathrm{l}$. The mean fetal/maternal ratio was 0.72 (SD 0.36). This ratio was higher $(p<0.05)$ during repeated $(n=14,0.8$, SD 0.37 ) compared to single ( $n=19,0.6$, SD 0.31$)$ dose administration.

Figure 1 illustrates the time-concentrations points collected in maternal and umbilical cord blood after single clindamycin $(900 \mathrm{mg})$ administration in 14 cases from 2 different studies. The pattern suggests that maternal-fetal transfer is fast, but incomplete.

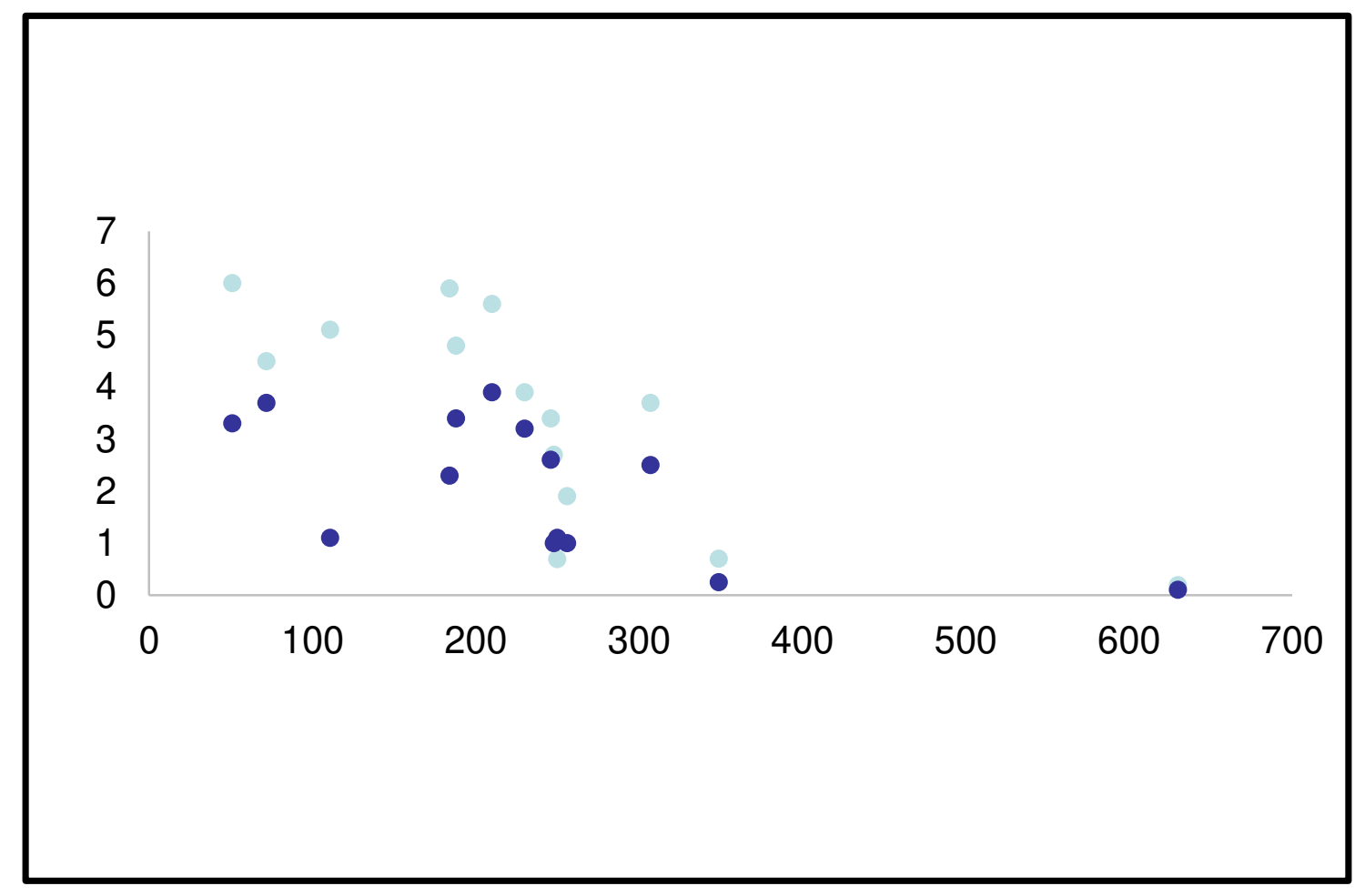

\section{Conclusions}

The pattern suggests that maternal-fetal transfer for clindamycin is fast, but incomplete. Since EUCAST minimal inhibitory concentrations (MIC) breakpoints are $\leq 0.5 \mathrm{mg} / \mathrm{l}$ for staphylococci and streptococci (including group B streptococcus), $\leq 4 \mathrm{mg} / \mathrm{l}$ for gram-positive anaerobes or gram-negative anaerobes, the concentrations reached are sufficient for some of the pathogens involved in neonatal sepsis. 